\title{
Diagnosis of Fasciola Hepatica by Endoscopic Ultrasound
}

\section{R. Sotoudehmanesh, A. Yoonessi}

Digestive Diseases Research Center, Tehran University of Medical Sciences, Tehran, Iran

A 28-year-old woman was admitted for colicky abdominal pain. Mild eosinophilia

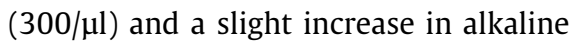
phosphatase were present. Transabdominal ultrasonography showed only mild dilation of the common bile duct. Figures $\mathbf{1}, \mathbf{2}$ show the endoscopic ultrasound (EUS) and endoscopic retrograde cholangiopancreatography (ERCP) findings, respectively. Parasitology confirmed the diagnosis of Fasciola hepatica. Endoscopic ultrasound may be helpful in the diagnosis of parasites in the biliary tract.

\section{Corresponding Author}

\section{R. Sotoudehmanesh, M.D.}

Digestive Disease Research Center, Shariati Hospital

North Kargar Avenue

14114 Tehran

Iran

Fax: + +98-21-8012992

E-mail: setoodeh@ams.ac.ir

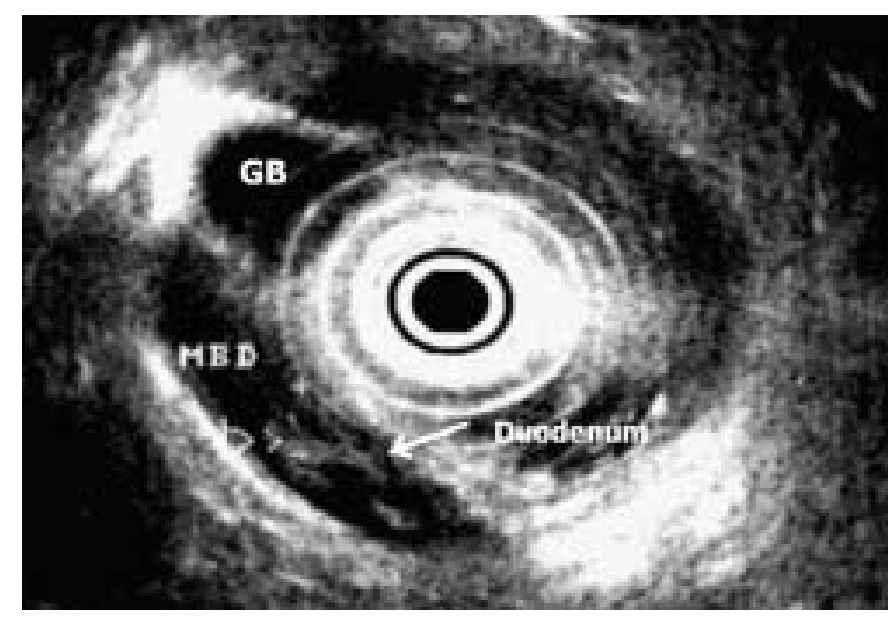

Figure 1 Endoscopic ultrasound revealed a spiralshaped mobile membrane (arrow) within the main bile duct (MBD). GB: gallbladder.

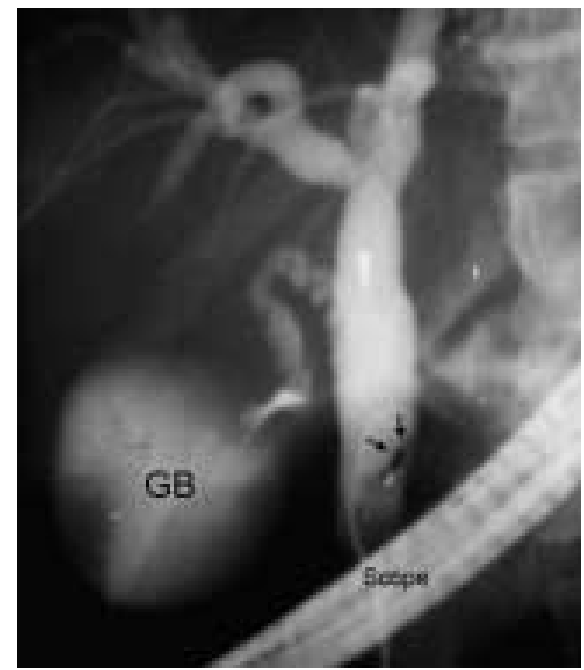

Figure 2 Endoscopic retrograde cholangiopancreatography showed a comma-shaped filling defect (arrows), and a live flatworm was extracted. GB: gallbladder. 